\title{
Linearized Form of the Generic Affine-Virasoro Action 1
}

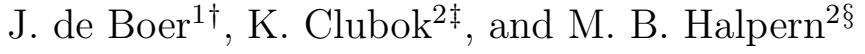 \\ 1. Institute for Theoretical Physics \\ State University of New York at Stony Brook \\ Stony Brook, New York 11794-3840, USA \\ 2. Department of Physics \\ University of California at Berkeley \\ and \\ Theoretical Physics Group \\ Lawrence Berkeley Laboratory \\ Berkeley, CA 94720, U.S.A.
}

\begin{abstract}
Halpern and Yamron have given a Lorentz, conformal, and Diff $\mathrm{S}_{2^{-}}$ invariant world-sheet action for the generic irrational conformal field theory, but the action is highly non-linear. In this paper, we introduce auxiliary fields to find an equivalent linearized form of the action, which shows in a very clear way that the generic affine-Virasoro action is a Diff $\mathrm{S}_{2}$-gauged WZW model. In particular, the auxiliary fields transform under Diff $\mathrm{S}_{2}$ as local Lie $g \times$ Lie $g$ connections, so that the linearized affine-Virasoro action bears an intriguing resemblance to the usual (Lie algebra) gauged WZW model.
\end{abstract}

*The work of $\mathrm{MBH}$ was supported in part by the Director, Office of Energy Research, Office of High Energy and Nuclear Physics, Division of High Energy Physics of the U.S. Department of Energy under Contract DE-AC03-76SF00098 and in part by the National Science Foundation under grant PHY90-21139. The work of JdB was sponsored in part by the National Science Foundation under grant PHY93-09888.

${ }^{\dagger} \mathrm{e}-\mathrm{mail}:$ DEBOER@MAX.PHYSICS.SUNYSB.EDU

‡e-mail: CLUBOK@PHYSICS.BERKELEY.EDU

§e-mail: MBHALPERN@LBL.GOV 


\section{Introduction}

It is now understood that affine Lie algebra $[1,2]$ underlies rational conformal field theory and irrational conformal field theory (ICFT), which includes rational conformal field theory as a small subspace.

The chiral stress tensors of ICFT are given by the general affine-Virasoro construction $[3,4]$

$$
T=L^{a b}{ }_{*}^{*} J_{a} J_{b}{ }_{*}^{*}
$$

where $J_{a}, a=1 \ldots \operatorname{dim} \mathrm{g}$ are the currents of affine $\mathrm{g}$ and $L^{a b}$ is a solution of the Virasoro master equation $[3,4]$. See references $[5,6]$ for reviews of recent developments in the Virasoro master equation and the associated affine-Virasoro Ward identities for the correlators of ICFT [7-9].

In this paper, we focus on the generic affine-Virasoro action [10], which is a Lorentz, conformal, and Diff $\mathrm{S}_{2}$-invariant world-sheet action for the generic ICFT. This action exhibits an elegant underlying geometry associated with the embedding of Diff $\mathrm{S}_{2}$ in (classical) affine $G \times G$, but the form of the action is highly non-linear.

The central result of this paper is an equivalent linearized form of this action, given in eq.(4.7), which shows in a very clear way that the generic affineVirasoro action is a Diff $\mathrm{S}_{2}$-gauged WZW model. In this form, the action bears an intriguing resemblance to the ordinary (Lie algebra) gauged WZW model $[11,12]$, and we will see that this resemblance is a consequence of the underlying geometry.

To understand the forms of the generic affine-Virasoro action, we need some basic facts about affine-Virasoro constructions.

The original affine-Virasoro action follows from the basic quantum hamiltonian! of the general theory $L$,

$$
H_{0}=L_{0}+\bar{L}_{0}=L^{a b}\left({ }_{*}^{*} J_{a} J_{b}+\bar{J}_{a} \bar{J}_{b}{ }_{*}^{*}\right)_{0}
$$

\footnotetext{
${ }^{\dagger}$ A natural off-critical extension of the general affine-Virasoro construction is defined by the hamiltonian $(1.2)$ with arbitrary $L^{a b}$. These theories are scale invariant and generically non-relativistic, generally achieving Lorentz invariance only at the conformal points $L^{a b}$ which solve the Virasoro master equation.
} 
where the barred currents $\bar{J}$ and Virasoro operator $\bar{T}=L^{a b}{ }_{*}^{*} \bar{J}_{a} \bar{J}_{b}{ }_{*}^{*}$ are rightmover copies of the left-movers $J$ and $T$. In general, the basic hamiltonian in (1.2) admits a local gauge invariance, described by the (symmetry) algebra of the commutant of $H_{0}$, and the physical (gauge-fixed) Hilbert space of the $L$ theory may be taken as the primary states under the symmetry algebra.

As a simple example, consider the stress tensor $T_{g / h}=L_{g / h}^{a b}{ }_{*}^{*} J_{a} J_{b}{ }_{*}^{*}$ of the $g / h$ coset constructions $[2,13,14]$, whose symmetry algebra is the affine algebra of $h$. Then the physical Hilbert space of the non-chiral coset construction may be chosen as the set of states

$$
\left.\left.J_{a}{ }^{m>0} \mid \text { phys }\right\rangle=\bar{J}_{a}{ }^{m>0} \mid \text { phys }\right\rangle=0, \quad a \in h
$$

which are primary under affine $h \times h$.

In the space of all conformal field theories, the coset constructions are only special points of higher symmetry. The symmetry algebra of the generic stress tensor $T$ is the Virasoro algebra of its commuting $K$-conjugate theory $\tilde{T}$ $[2,11,12,3]$,

$$
\tilde{T}=\tilde{L}^{a b}{ }_{*}^{*} J_{a} J_{b}{ }_{*}^{*}=T_{g}-T, \quad \tilde{L}^{a b}=L_{g}^{a b}-L^{a b}, \quad c(\tilde{L})=c_{g}-c(L)
$$

where $T_{g}$ is the affine-Sugawara construction on $\mathrm{g}[2,13,15,16]$ and $c_{g}$ is its central charge. Then the physical Hilbert space of the generic theory $L$ may be chosen as the set of states

$$
\begin{gathered}
\left.\left.\tilde{L}^{m>0} \mid \text { phys }\right\rangle=\overline{\tilde{L}}^{m>0} \mid \text { phys }\right\rangle=0 \\
\tilde{T}=\tilde{L}^{a b}{ }_{*}^{*} J_{a} J_{b}{ }_{*}^{*}, \quad \overline{\tilde{T}}=\tilde{L}_{*}^{a b}{ }_{*}^{*} \bar{J}_{a} \bar{J}_{b}{ }_{*}^{*}
\end{gathered}
$$

which are Virasoro primary under the $K$-conjugate stress tensors $\tilde{T}$ and $\overline{\tilde{T}}$.

Transcribing (1.3) and (11.5) into the language of world-sheet actions, we understand that we will obtain a spin-1 gauge theory [11,12] for the special cases of the coset constructions and a spin-2 gauge theory [10] for the generic ICFT, where the generic theory $L$ is gauged by its $K$-conjugate theory $\tilde{L}$.

One other fact about the Virasoro master equation is necessary to understand the generic affine-Virasoro action. Since an action begins as a semiclassical description of a quantum system, the affine-Virasoro action involves 
the semi-classical (or high-level) form of the $K$-conjugate pairs of solutions of the master equation $[17,10]$,

$$
\begin{gathered}
L_{\infty}^{a b}=\frac{1}{2} G^{a c} P_{c}^{b}, \quad \tilde{L}_{\infty}^{a b}=\frac{1}{2} G^{a c} \tilde{P}_{c}^{b}, \quad L_{\infty}^{a b}+\tilde{L}_{\infty}^{a b}=L_{g, \infty}^{a b}=\frac{1}{2} G^{a b} \\
c\left(L_{\infty}\right)=\operatorname{dim} P, \quad c\left(\tilde{L}_{\infty}\right)=\operatorname{dim} \tilde{P}, \quad c\left(L_{\infty}\right)+c\left(\tilde{L}_{\infty}\right)=c\left(L_{g, \infty}\right)=\operatorname{dim} g \\
P^{2}=P, \quad \tilde{P}^{2}=\tilde{P}, \quad P+\tilde{P}=1, \quad P \tilde{P}=\tilde{P} P=0 .
\end{gathered}
$$

Here, $P$ and $\tilde{P}$ are the high-level projectors of the $L$ and the $\tilde{L}$ theories respectively, whose high-level central charges are the dimensions of their projectors. $G^{a b}$ is the inverse of $G_{a b}$, which is the coefficient of the central term in the general affine algebra. The results in (1.6) are valid for high levels $k_{I}$ of $g=\oplus_{I} g_{I}$, but the reader may wish to think in terms of simple g, for which $G^{a b}=k^{-1} \eta^{a b}$ where $\eta^{a b}$ is the inverse Killing metric of $g$.

Although all high-level smooth solutions of the master equation have the form (1.6), it is important to recall that not all projectors are solutions $[17,10]$. This is the high-level classification problem of ICFT, which has been partially solved in terms of graph theory and generalized graph theory $[6,18]$. In the graph theories, the projectors are adjacency matrices of the graphs, each of which labels a level family of ICFT's.

\section{Notation and the WZW Model}

We begin on the group manifold $G$ with $g(x) \in G$ in matrix representation $T$,

$$
\left[T_{a}, T_{b}\right]=i f_{a b}^{c}, \quad \operatorname{tr}\left(T_{a} T_{b}\right)=y G_{a b}, \quad a, b, c=1, \ldots, \operatorname{dim} g
$$

and a set of coordinates $x^{i}(\sigma, \tau), i=1, \ldots$, dim g. Introduce the left-invariant and right-invariant vielbeins $e_{i}, \bar{e}_{i}$ and the antisymmetric tensor field $B_{i j}$ by

$$
\begin{gathered}
e_{i} \equiv-i g^{-1} \partial_{i} g=e_{i}{ }^{a} T_{a} \quad \bar{e}_{i} \equiv-i g \partial_{i} g^{-1}=\bar{e}_{i}{ }^{a} T_{a} \\
\partial_{[i} e_{j]}^{a}=e_{i}{ }^{b} e_{j}{ }^{c} f_{b c}{ }^{a}, \quad \partial_{[i} \bar{e}_{j]}^{a}=\bar{e}_{i}{ }^{b} \bar{e}_{j}^{c} f_{b c}{ }^{a} \\
i \operatorname{tr}\left(e_{i}\left[e_{j}, e_{k}\right]\right)=-i \operatorname{tr}\left(\bar{e}_{i}\left[\bar{e}_{j}, \bar{e}_{k}\right]\right)=\partial_{i} B_{j k}+\partial_{j} B_{k i}+\partial_{k} B_{i j} .
\end{gathered}
$$

We also introduce inverse vielbeins $e_{a}{ }^{i}$ and $\bar{e}_{a}{ }^{i}$. 
In this notation, the WZW action [15] is given by,

$$
\begin{gathered}
S_{W Z W}=\int d \tau d \sigma\left(\mathcal{L}_{W Z W}+\Gamma\right) \\
\mathcal{L}_{W Z W}=\frac{1}{8 \pi} G_{a b} e_{i}{ }^{a} e_{j}{ }^{b}\left(\dot{x}^{i} \dot{x}^{j}-x^{i} x^{\prime j}\right), \quad \Gamma=\frac{1}{4 \pi y} B_{i j} \dot{x}^{i} x^{\prime j}
\end{gathered}
$$

where $\Gamma$ is the WZW term. The corresponding classical WZW hamiltonian is

$$
\begin{gathered}
H_{W Z W}=\int_{0}^{2 \pi} d \sigma \mathcal{H}_{W Z W} \\
\mathcal{H}_{W Z W}=\frac{1}{2 \pi} L_{g, \infty}^{a b}\left(J_{a} J_{b}+\bar{J}_{a} \bar{J}_{b}\right), \quad L_{g, \infty}^{a b}=\frac{1}{2} G^{a b}
\end{gathered}
$$

where $L_{g, \infty}^{a b}$ is the high-level form of the affine-Sugawara construction on $g$, and the currents $J_{a}, \bar{J}_{a}, a=1, \ldots, \operatorname{dim} g$ are given by the canonical representation |19,

$$
\begin{gathered}
J_{a}=2 \pi e_{a}^{i} \hat{p}_{i}+\frac{1}{2} G_{a b} e_{i}{ }^{b} x^{i}, \quad \bar{J}_{a}=2 \pi \bar{e}_{a}^{i} \hat{p}_{i}-\frac{1}{2} G_{a b} \bar{e}_{i}{ }^{b} x^{i} \\
\hat{p}_{i} \equiv p_{i}-\frac{1}{4 \pi y} B_{i j} x^{j}
\end{gathered}
$$

which satisfies the bracket (classical) form of affine $g \times g$.

\section{The Generic Affine-Virasoro Action}

Assuming that the currents satisfy the algebra of (classical) affine $g \times g$, we have the classical affine-Virasoro hamiltonian [10],

$$
\begin{gathered}
H=\int_{0}^{2 \pi} d \sigma \mathcal{H} \\
\mathcal{H}=\mathcal{H}_{0}\left(L_{\infty}\right)+v \cdot K\left(\tilde{L}_{\infty}\right) \\
\mathcal{H}_{0}\left(L_{\infty}\right)=\frac{1}{2 \pi} L_{\infty}^{a b}\left(J_{a} J_{b}+\bar{J}_{a} \bar{J}_{b}\right), \quad v \cdot K\left(\tilde{L}_{\infty}\right)=\frac{1}{2 \pi} \tilde{L}_{\infty}^{a b}\left(v J_{a} J_{b}+\bar{v} \bar{J}_{a} \bar{J}_{b}\right) .
\end{gathered}
$$

for the generic theory $L$, gauged by its $K$-conjugate theory $\tilde{L}$. Note that all four commuting (classical) Virasoro operators (the stress tensors),

$$
\frac{1}{2 \pi} L_{\infty}^{a b} J_{a} J_{b}, \quad \frac{1}{2 \pi} L_{\infty}^{a b} \bar{J}_{a} \bar{J}_{b}, \quad \frac{1}{2 \pi} \tilde{L}_{\infty}^{a b} J_{a} J_{b}, \quad \frac{1}{2 \pi} \tilde{L}_{\infty}^{a b} \bar{J}_{a} \bar{J}_{b}
$$

are involved here, as in the quantum theory, with the semiclassical substitution $L \rightarrow L_{\infty}, \tilde{L} \rightarrow \tilde{L}_{\infty}$. The first two stress tensors are used to form the classical basic hamiltonian $H_{0}=\int d \sigma \mathcal{H}_{0}$ of the $L$ theory, while the commuting $K$-conjugate 
stress tensors (of the $\tilde{L}$ theory) play the role of Gauss' law, and must be added, as shown, with the spin-2 gauge field $v, \bar{v}$.

For passage to the action, one needs to choose a canonical representation of the currents. Following Ref.[10], we choose the representation in (2.5), but it should be emphasized that other canonical representations can be chosen [20] which lead to presumably equivalent actions.

With (2.5), one obtains the hamiltonian equation of motion,

$$
\begin{gathered}
\dot{x}^{i}=4 \pi e_{a}{ }^{i} G^{a c} M_{c}{ }^{b} e_{b}{ }^{j} \hat{p}_{j}+e_{a}{ }^{i} G^{a b} N_{b}{ }^{c} G_{c d} e_{j}{ }^{d} x^{j} \\
M \equiv 1-\frac{1-v}{2} \tilde{P}-\frac{1-\bar{v}}{2} \omega \tilde{P} \omega^{-1}, \quad N \equiv-\frac{1-v}{2} \tilde{P}+\frac{1-\bar{v}}{2} \omega \tilde{P} \omega^{-1} \\
g T_{a} g^{-1}=\omega_{a}^{b} T_{b}, \quad \omega_{a}{ }^{c} G_{c d} \omega_{b}{ }^{d}=G_{a b} \\
\bar{e}_{i}{ }^{a}=-e_{i}{ }^{b} \omega_{b}{ }^{a}, \quad \bar{e}_{a}{ }^{i}=-\left(\omega^{-1}\right)_{a}{ }^{b} e_{b}{ }^{i}
\end{gathered}
$$

where $\omega_{a}^{b}$ is the adjoint action of $g$. Eliminating $p$ in favor of $\dot{x}$, one finds the affine-Virasoro action of the generic theory $L$ [10],

$$
\begin{gathered}
S=\int d \tau d \sigma(\mathcal{L}+\Gamma) \\
\mathcal{L}=\frac{1}{8 \pi} e_{i}^{a} G_{b c} e_{j}^{c}\left[\left[f(Z)+\alpha \bar{\alpha} \omega \tilde{P} \omega^{-1} f(Z) \tilde{P}\right]_{a}^{b}\left(\dot{x}^{i} \dot{x}^{j}-x^{i} x^{j}\right)\right. \\
+\alpha[f(Z) \tilde{P}]_{a}^{b}\left(\dot{x}^{i} \dot{x}^{j}+x^{i} x^{j}+\dot{x}^{(i} x^{j)^{\prime}}\right) \\
+\bar{\alpha}\left[\omega \tilde{P} \omega^{-1} f(Z)\right]_{a}^{b}\left(\dot{x}^{i} \dot{x}^{j}+x^{i} x^{j}-\dot{x}^{(i} x^{j) \prime}\right) \\
\left.+\left[1-f(Z)+\alpha \bar{\alpha} \omega \tilde{P} \omega^{-1} f(Z) \tilde{P}\right]_{a}^{b}\left(\dot{x}^{[i} x^{j] \prime}\right)\right] \\
f(Z) \equiv[1-\alpha \bar{\alpha} Z]^{-1}, \quad Z \equiv \tilde{P} \omega \tilde{P} \omega^{-1}, \quad \alpha \equiv \frac{1-v}{1+v}, \quad \bar{\alpha} \equiv \frac{1-\bar{v}}{1+\bar{v}} .
\end{gathered}
$$

The Lorentz, diffeomorphism, and conformal symmetries of this action are discussed in the original reference, and we confine ourselves here to some brief remarks which will be useful below.

A. WZW limits. The affine-Virasoro action reduces to the WZW action $S_{W Z W}$ when we choose $L_{\infty}^{a b}=L_{g, \infty}^{a b}$.

For any $L_{\infty}^{a b}$, the action also reduces to $S_{W Z W}$ in the WZW gauge,

$$
v=\bar{v}=1, \quad \alpha=\bar{\alpha}=0 .
$$


The hamiltonian theory in this gauge must be taken with the $K$-conjugate constraints $\tilde{L}_{\infty}^{a b} J_{a} J_{b}=\tilde{L}_{\infty}^{a b} \bar{J}_{a} \bar{J}_{b}=0$, so that $\mathcal{H} \sim \mathcal{H}_{0}\left(L_{\infty}\right)$ on the constrained space. B. Diff $\mathrm{S}_{2}(K)$ invariance. The affine-Virasoro action is invariant under the Diff $\mathrm{S}_{2}(K)$ coordinate transformations,

$$
\begin{gathered}
\delta x^{i}=\Lambda^{a} e_{a}{ }^{i}+\bar{\Lambda}^{a} \bar{e}_{a}{ }^{i} \\
\delta g=g i \Lambda^{a} T_{a}-i \bar{\Lambda}^{a} T_{a} g \\
\delta J_{a}=f_{a b}{ }^{c} \Lambda^{b} J_{c}+G_{a b} \partial_{\sigma} \Lambda^{b}, \quad \delta \bar{J}_{a}=f_{a b}{ }^{b} \bar{\Lambda}^{b}-G_{a b} \partial_{\sigma} \bar{\Lambda}^{b} \\
\Lambda^{a}=2 \epsilon \tilde{L}_{\infty}^{a b} J_{b}, \quad \bar{\Lambda}^{a}=2 \bar{\epsilon} \tilde{L}_{\infty}^{a b} \bar{J}_{b} \\
\delta v=\dot{\epsilon}+\epsilon \stackrel{\leftrightarrow}{\partial}_{\sigma} v, \quad \delta \bar{v}=\dot{\bar{\epsilon}}+\bar{v} \stackrel{\leftrightarrow}{\partial}_{\sigma} \bar{\epsilon}
\end{gathered}
$$

generated by the stress tensors of the $K$-conjugate theory.

With Ref. [10], we remark on the embedding of Diff $\mathrm{S}_{2}(K)$ in (classical) affine $G \times G$. The transformation of the group element $g$ in eq. (3.6b) shows that infinitesimal Diff $\mathrm{S}_{2}(K)$ transformations are particular transformations in (classical) affine $G \times G$, with the current-dependent local Lie $g \times$ Lie $g$ gauge parameters $\Lambda, \bar{\Lambda}$ in eq.(3.6d). In this sense, Diff $\mathrm{S}_{2}(K)$ is embedded locally in (classical) affine $G \times G$. Moreover, the result (3.6c) shows that the currents $J, \bar{J}$ transform under Diff $\mathrm{S}_{2}(K)$ as local Lie $g \times$ Lie $g$ gauge fields, or connections, with the same gauge parameters $\Lambda, \bar{\Lambda}$. The embedding of Diff $S_{2}$ in the affine algebra is the underlying geometry of the generic affine-Virasoro construction, and this geometry will continue to play a central role in the linearized action below.

The transformation of $v, \bar{v}$ in (3.6e) allows the identification of a second-rank tensor field,

$$
\tilde{h}_{m n} \equiv e^{-\phi}\left(\begin{array}{cc}
-v \bar{v} & \frac{1}{2}(v-\bar{v}) \\
\frac{1}{2}(v-\bar{v}) & 1
\end{array}\right), \quad \sqrt{-\tilde{h}} \tilde{h}^{m n}=\frac{2}{v+\bar{v}}\left(\begin{array}{cc}
-1 & \frac{1}{2}(v-\bar{v}) \\
\frac{1}{2}(v-\bar{v}) & v \bar{v}
\end{array}\right)
$$

which was called the $K$-conjugate metric in Ref.[10]. Note that in the WZW gauge,

$$
\sqrt{-\tilde{h}} \tilde{h}^{m n}=\left(\begin{array}{cc}
-1 & 0 \\
0 & 1
\end{array}\right)
$$

so that the WZW gauge is the conformal gauge of the generic action. 
To identify the $K$-conjugate metric more precisely, we define the symmetric stress tensor,

$$
\Theta^{m n}=\frac{2}{\sqrt{-\tilde{h}}} \frac{\delta S_{A V}}{\delta \tilde{h}_{m n}}
$$

which is covariantly conserved. In the WZW gauge, we find that

$$
\begin{aligned}
& \Theta_{00}=\Theta_{11}=\frac{1}{2 \pi} \tilde{L}_{\infty}^{a b}\left(J_{a} J_{b}+\bar{J}_{a} \bar{J}_{b}\right) \\
& \Theta_{01}=\Theta_{10}=\frac{1}{2 \pi} \tilde{L}_{\infty}^{a b}\left(J_{a} J_{b}-\bar{J}_{a} \bar{J}_{b}\right)
\end{aligned}
$$

which identifies the $K$-conjugate metric $\tilde{h}_{m n}$ as the world-sheet metric of the $\tilde{L}$ theory.

C. Rigid conformal invariance. The action is invariant under a rigid (ungauged) conformal invariance, including a rigid world-sheet Lorentz invariance, of the usual form,

$$
\begin{aligned}
& \delta \xi^{ \pm}=-\rho^{ \pm}\left(\xi^{ \pm}\right), \quad \delta x^{i}=\left(\rho^{+} \partial_{+}+\rho^{-} \partial_{-}\right) x^{i} \\
& \delta \alpha=\left(\rho^{+} \partial_{+}+\rho^{-} \partial_{-}\right) \alpha+\left(\partial_{-} \rho^{-}-\partial_{+} \rho^{+}\right) \alpha \\
& \delta \bar{\alpha}=\left(\rho^{+} \partial_{+}+\rho^{-} \partial_{-}\right) \bar{\alpha}+\left(\partial_{+} \rho^{+}-\partial_{-} \rho^{-}\right) \bar{\alpha}
\end{aligned}
$$

where we have defined $\xi^{ \pm} \equiv(\tau \pm \sigma) / \sqrt{2}$. The $\alpha, \bar{\alpha}$ transformations in (3.11) are equivalent to the $v, \bar{v}$ transformations in eq.(4.12) of Ref. [10], and identify $\alpha$ and $\bar{\alpha}$ as $(-1,1)$ and $(1,-1)$ conformal tensors respectively. With these identifications, each term in the action density (3.4b) is $(1,1)$ on inspection.

The rigid conformal group is the conformal group of the $L$ theory, generated by the stress tensors $L_{\infty}^{a b} J_{a} J_{b} / 2 \pi$ and $L_{\infty}^{a b} \bar{J}_{a} \bar{J}_{b} / 2 \pi$. The theory also has a gauged conformal group [10], in Diff $\mathrm{S}_{2}(K)$, associated to the $K$-conjugate stress tensors $\tilde{L}_{\infty}^{a b} J_{a} J_{b} / 2 \pi$ and $\tilde{L}_{\infty}^{a b} \bar{J}_{a} \bar{J}_{b} / 2 \pi$.

\section{Linearized Form of the Affine-Virasoro Action}

The affine-Virasoro action in (3.4) is highly non-linear, but we may linearize it by the introduction of auxiliary fields. 
We begin by defining two matrices,

$$
\begin{aligned}
W_{a}{ }^{b} & \equiv \delta_{a}{ }^{b}+\alpha \tilde{P}_{a}^{b}, & \left(W^{-1}\right)_{a}{ }^{b} & =\delta_{a}{ }^{b}+\frac{1}{2}(v-1) \tilde{P}_{a}{ }^{b} \\
\bar{W}_{a}{ }^{b} & \equiv \delta_{a}{ }^{b}+\bar{\alpha} \tilde{P}_{a}{ }^{b}, & \left(\bar{W}^{-1}\right)_{a}{ }^{b} & =\delta_{a}{ }^{b}+\frac{1}{2}(\bar{v}-1) \tilde{P}_{a}{ }^{b}
\end{aligned}
$$

where $\alpha, \bar{\alpha}$ are defined in eq.(3.4). Using these matrices, we add two terms to the hamiltonian and the corresponding action of the theory,

$$
\begin{aligned}
\mathcal{H}^{\prime}=\mathcal{H}+\Delta \mathcal{H}, \quad S^{\prime}=\int d \tau d \sigma\left[p_{i} \dot{x}^{i}-\mathcal{H}-\Delta \mathcal{H}\right] \\
\Delta \mathcal{H}=-\frac{1}{2 \pi}\left(B_{a}-\left(W^{-1}\right)_{a}{ }^{d} J_{d}\right) G^{a c} W_{c}^{b}\left(B_{b}-\left(W^{-1}\right)_{b}{ }^{d} J_{d}\right) \\
-\frac{1}{2 \pi}\left(\bar{B}_{a}-\left(\bar{W}^{-1}\right)_{a}{ }^{d} \bar{J}_{d}\right) G^{a c} \bar{W}_{c}^{b}\left(\bar{B}_{b}-\left(\bar{W}^{-1}\right)_{b}{ }^{d} \bar{J}_{d}\right) .
\end{aligned}
$$

Here, $B_{a}$ and $\bar{B}_{a}, a=1, \ldots, \operatorname{dim} g$ are a set of auxiliary fields, called the first set of auxiliary connections for reasons discussed below. Since the action is quadratic in $B$ and $\bar{B}$, the auxiliary connections are easily integrated out, showing that the theory is unchanged for averages of functions of $x$ and $p$.

For passage to the action, it is convenient to rearrange $H^{\prime}$ as follows,

$$
\begin{aligned}
\mathcal{H}^{\prime}= & \mathcal{H}_{W Z W} \\
& -\frac{\alpha}{\pi} \tilde{L}_{\infty}^{a b} B_{a} B_{b}-\frac{1}{2 \pi}\left(B_{a}-J_{a}\right) G^{a b}\left(B_{b}-J_{b}\right) \\
& -\frac{\bar{\alpha}}{\pi} \tilde{L}_{\infty}^{a b} \bar{B}_{a} \bar{B}_{b}-\frac{1}{2 \pi}\left(\bar{B}_{a}-\bar{J}_{a}\right) G^{a b}\left(\bar{B}_{b}-\bar{J}_{b}\right) \\
\dot{x}^{i}= & -4 \pi e_{a}{ }^{i} G^{a b} e_{b}{ }^{j} \hat{p}_{j}+2 e_{a}{ }^{i} G^{a b}\left(B_{b}-\omega_{b}{ }^{c} \bar{B}_{c}\right) .
\end{aligned}
$$

Because of the simple equation of motion in (4.3b), we obtain our first linearized form of the affine-Virasoro action,

$$
\begin{gathered}
S^{\prime}=\int d \tau d \sigma\left(\mathcal{L}^{\prime}+\Gamma\right) \\
\mathcal{L}^{\prime}=-\frac{1}{8 \pi} G_{a b}\left(e_{\tau}^{a} e_{\tau}^{b}-e_{\sigma}^{a} e_{\sigma}^{b}\right)+\frac{1}{\pi} G^{a b} B_{a} \omega_{b}^{c} \bar{B}_{c} \\
+\frac{\alpha}{\pi} \tilde{L}_{\infty}^{a b} B_{a} B_{b}+\frac{1}{2 \pi}\left(e_{\tau}^{a}-e_{\sigma}^{a}\right) B_{a} \\
+\frac{\bar{\alpha}}{\pi} \tilde{L}_{\infty}^{a b} \bar{B}_{a} \bar{B}_{b}+\frac{1}{2 \pi}\left(\bar{e}_{\tau}^{a}+\bar{e}_{\sigma}^{a}\right) \bar{B}_{a}
\end{gathered}
$$


where we have introduced $e_{\tau}^{a} \equiv e_{i}{ }^{a} \dot{x}^{i}, e_{\sigma}{ }^{a} \equiv e_{i}{ }^{a} x^{i}$ and similarly for $\bar{e}$. Note that the first term in $\mathcal{L}^{\prime}$ and the WZW term $\Gamma$ almost comprise the action $S_{W Z W}$, but the kinetic energy term has the wrong sign.

To correct this sign, we introduce a second set of auxiliary connections,

$$
A_{a} \equiv\left(\omega^{-1}\right)_{a}^{b}\left(B_{b}-\frac{1}{2} G_{b c}\left(e_{\tau}^{c}+e_{\sigma}^{c}\right)\right), \quad \bar{A}_{a} \equiv \omega_{a}^{b}\left(\bar{B}_{b}-\frac{1}{2} G_{b c}\left(\bar{e}_{\tau}^{c}-\bar{e}_{\sigma}{ }^{c}\right)\right)
$$

with $a=1, \ldots, \operatorname{dim} g$. In terms of these auxiliary fields, we obtain the final form of the linearized affine-Virasoro action,

$$
\begin{gathered}
S^{\prime}=S_{W Z W}+\int d \tau d \sigma \Delta \mathcal{L} \\
\Delta \mathcal{L}=\frac{\alpha}{\pi} \tilde{L}_{\infty}^{a b}\left(\omega_{a}^{c} A_{c}+\frac{1}{2} G_{a c}\left(e_{\tau}^{c}+e_{\sigma}^{c}\right)\right)\left(\omega_{b}{ }^{d} A_{d}+\frac{1}{2} G_{b d}\left(e_{\tau}{ }^{d}+e_{\sigma}{ }^{d}\right)\right) \\
+\frac{\bar{\alpha}}{\pi} \tilde{L}_{\infty}^{a b}\left(\left(\omega^{-1}\right)_{a}{ }^{c} \bar{A}_{c}+\frac{1}{2} G_{a c}\left(\bar{e}_{\tau}^{c}-\bar{e}_{\sigma}{ }^{c}\right)\right)\left(\left(\omega^{-1}\right)_{b}{ }^{d} \bar{A}_{d}+\frac{1}{2} G_{b d}\left(\bar{e}_{\tau}{ }^{d}-\bar{e}_{\sigma}{ }^{d}\right)\right) \\
+\frac{1}{\pi} \bar{A}_{a} G^{a b} \omega_{b}^{c} A_{c}
\end{gathered}
$$

where $S_{W Z W}$ is the WZW action in (2.3). In this form, we may easily verify that $S^{\prime}$ reduces to $S_{W Z W}$ in the WZW gauge, since the auxiliary fields essentially decouple.

For the reader's convenience, we also give this action in terms of the group variable $g$,

$$
\begin{gathered}
S^{\prime}=S_{W Z W}+\int d^{2} z \Delta \mathcal{L} \\
S_{W Z W}=-\frac{1}{2 \pi y} \int d^{2} z \operatorname{tr}\left(g^{-1} \partial g g^{-1} \bar{\partial} g\right)-\frac{1}{12 \pi y} \int_{M} \operatorname{tr}\left(g^{-1} d g\right)^{3} \\
\Delta \mathcal{L}=-\frac{\alpha}{\pi y^{2}} \tilde{L}_{\infty}^{a b} \operatorname{tr}\left(T_{a} g^{-1} D g\right) \operatorname{tr}\left(T_{b} g^{-1} D g\right) \\
-\frac{\bar{\alpha}}{\pi y^{2}} \tilde{L}_{\infty}^{a b} \operatorname{tr}\left(T_{a} g \bar{D} g^{-1}\right) \operatorname{tr}\left(T_{b} g \bar{D} g^{-1}\right) \\
+\frac{1}{\pi y} \operatorname{tr}\left(g \bar{A} g^{-1} A\right) \\
\bar{A} \equiv A_{a} G^{a b} T_{b}, \quad \partial \equiv \frac{1}{2}\left(\partial_{\tau}+\partial_{\sigma}\right), \quad \bar{\partial} \equiv \frac{1}{2}\left(\partial_{\tau}-\partial_{\sigma}\right) \\
D \equiv \partial+i A, \quad \bar{D} \equiv \bar{\partial}+i \bar{A}
\end{gathered}
$$


where we have defined the covariant derivatives $D, \bar{D}$ and $d^{2} z=d \tau d \sigma$.

In this form, the theory is clearly seen as a Diff $\mathrm{S}_{2}$-gauged WZW model, which bears an intriguing resemblance to the form of the usual (Lie algebra) gauged WZW model $[11,12]$. As we shall see in the following section, this resemblance is due to the Diff $\mathrm{S}_{2}(K)$ transformations of the auxiliary connections.

It is straightforward to check that the linearized action is equivalent to the non-linear form of the affine-Virasoro action. Using the inversion formula

$$
\begin{array}{cc}
C_{A}{ }^{B}=\left(\begin{array}{cc}
\alpha \tilde{P}_{a}^{b} & \delta_{a}^{b} \\
\delta_{a}^{b} & \bar{\alpha}\left(\omega \tilde{P} \omega^{-1}\right)_{a}{ }^{b}
\end{array}\right), & A, B=1, \ldots, 2 \operatorname{dim} g \\
\left(C^{-1}\right)_{A}{ }^{B} & =\left(\begin{array}{cc}
-\bar{\alpha}\left(\omega \tilde{P} \omega^{-1} f(Z)\right)_{a}{ }^{b} & \left(1+\alpha \bar{\alpha} \omega \tilde{P} \omega^{-1} f(Z) \tilde{P}\right)_{a}{ }^{b} \\
f(Z)_{a}{ }^{b} & -\alpha(f(Z) \tilde{P})_{a}{ }^{b}
\end{array}\right)
\end{array}
$$

to integrate out the auxiliary fields in (4.4) or (4.6), we obtain exactly (3.4) in the form

$$
\begin{gathered}
S=\int d \tau d \sigma(\mathcal{L}+\Gamma) \\
\mathcal{L}=-\frac{1}{8 \pi} G_{a b}\left(e_{\tau}^{a} e_{\tau}^{b}-e_{\sigma}^{a} e_{\sigma}^{b}\right)-\frac{1}{8 \pi} E^{A}\left(C^{-1}\right)_{A}{ }^{B} E_{B} \\
E^{A}=\left(\left(e_{\tau}^{a}-e_{\sigma}^{a}\right), \quad\left(\bar{e}_{\tau}^{a}+\bar{e}_{\sigma}^{a}\right)\right), \quad E_{B}=\left(\begin{array}{c}
G_{b c}\left(e_{\tau}^{c}-e_{\sigma}^{c}\right) \\
G_{b c}\left(\bar{e}_{\tau}{ }^{c}+\bar{e}_{\sigma}^{c}\right)
\end{array}\right) .
\end{gathered}
$$

\section{Invariances of the Linearized Action}

Because it is simpler, we discuss first the rigid conformal invariance, which is the conformal invariance of the $L$ theory. We find that the actions (4.4) and (4.6) or (4.7) are invariant under the transformations (3.11), supplemented by the conformal transformations of the auxiliary connections,

$$
\begin{gathered}
\delta B_{a}=\left(\rho^{+} \partial_{+}+\rho^{-} \partial_{-}\right) B_{a}+\partial_{+} \rho^{+} B_{a}, \quad \delta \bar{B}_{a}=\left(\rho^{+} \partial_{+}+\rho^{-} \partial_{-}\right) \bar{B}_{a}+\partial_{-} \rho^{-} B_{a} \\
\delta A=\left(\rho^{+} \partial_{+}+\rho^{-} \partial_{-}\right) A+\partial_{+} \rho^{+} A, \quad \delta \bar{A}=\left(\rho^{+} \partial_{+}+\rho^{-} \partial_{-}\right) \bar{A}+\partial_{-} \rho^{-} A
\end{gathered}
$$

which identify $A$ and $B$ as $(1,0)$ tensors and $\bar{A}$ and $\bar{B}$ as $(0,1)$ tensors. With

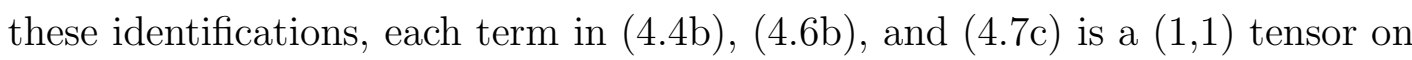
inspection. 
For Diff $\mathrm{S}_{2}(K)$, we find that the action (4.4) is invariant under

$$
\begin{gathered}
\delta x^{i}=\lambda^{a} e_{a}{ }^{i}+\bar{\lambda}^{a} \bar{e}_{a}{ }^{i} \\
\delta \alpha=-\bar{\partial} \xi+\xi \overleftrightarrow{\partial} \alpha, \quad \delta \bar{\alpha}=-\partial \bar{\xi}+\bar{\xi} \overleftrightarrow{\bar{\partial}} \bar{\alpha} \\
\delta B_{a}=f_{a b}{ }^{c} \lambda^{b} B_{c}+G_{a b} \partial \lambda^{b}, \quad \delta \bar{B}_{a}=f_{a b}{ }^{c} \bar{\lambda}^{b} \bar{B}_{c}+G_{a b} \bar{\partial} \bar{\lambda}^{b} \\
\lambda^{a} \equiv 2 \xi \tilde{L}_{\infty}^{a b} B_{b}, \quad \bar{\lambda}^{a} \equiv 2 \bar{\xi} \tilde{L}_{\infty}^{a b} \bar{B}_{b}
\end{gathered}
$$

where $\xi, \bar{\xi}$ are the diffeomorphism parameters. The $\alpha, \bar{\alpha}$ diffeomorphisms in (5.2b) are equivalent to the $v, \bar{v}$ diffeomorphisms in (3.6e) under the parametric redefinition

$$
\xi=(1+\alpha) \epsilon, \quad \bar{\xi}=(1+\bar{\alpha}) \bar{\epsilon}
$$

where $\epsilon, \bar{\epsilon}$ are the diffeomorphism parameters in (3.6).

The action (4.6) or (4.7) is also invariant under Diff $\mathrm{S}_{2}(K)$. For the vielbein form of the final action in (4.6), the transformation

$$
\delta A_{a}=f_{a b}{ }^{c} \bar{\lambda}^{b} A_{c}+G_{a b} \partial \bar{\lambda}^{b}, \quad \delta \bar{A}_{a}=f_{a b}{ }^{c} \lambda^{b} \bar{A}_{c}+G_{a b} \bar{\partial} \lambda^{b}
$$

replaces that of $B$ in (5.20). For the $g$ form of the final action in (4.7), the transformations

$$
\begin{gathered}
\delta g=g i \lambda-i \bar{\lambda} g \\
\delta A=\partial \bar{\lambda}+i[A, \bar{\lambda}], \quad \delta \bar{A}=\bar{\partial} \lambda+i[\bar{A}, \lambda] \\
\lambda \equiv \lambda^{a} T_{a}, \quad \bar{\lambda} \equiv \bar{\lambda}^{a} T_{a}
\end{gathered}
$$

replace those for $x$ and $B$ in (5.2). In (5.4) and (5.5), it is understood that the gauge parameters $\lambda^{a}(B(A))$ and $\bar{\lambda}^{a}(\bar{B}(\bar{A}))$ are expressed in terms of the connections $A$ and $\bar{A}$, using eqs.(5.2d) and the inverse of (4.5).

We remark in particular that the auxiliary connections in (5.2c), (5.4), and (5.5) transform under Diff $\mathrm{S}_{2}(K)$ as local Lie $g \times$ Lie $g$ connections, with fielddependent local Lie $g \times$ Lie $g$ parameters $\lambda, \bar{\lambda}$, restricted as shown in (5.2d). As emphasized above, and in Ref. [10], this is possible because Diff $\mathrm{S}_{2}(K)$ is locally embedded in (classical) affine $G \times G$. The Diff $\mathrm{S}_{2}(K)$ transformations of the auxiliary connections are also responsible for the intriguing resemblance of the 
linearized action (4.6) or (4.7) to the form of the usual (Lie algebra) gauged WZW model $[11,12]$.

Because verification of the diffeomorphism invariance of the linearized actions involves considerable algebra, we give some useful intermediate results for the $g$ form of the final action in (4.7). We need

$$
\delta\left(g^{-1} D g\right)=\partial(i \lambda)+\left[g^{-1} D g, i \lambda\right], \quad \delta\left(g \bar{D} g^{-1}\right)=\bar{\partial}(i \bar{\lambda})+\left[g \bar{D} g^{-1}, i \bar{\lambda}\right]
$$

and, using the explicit form of $\tilde{L}_{\infty}^{a b}$ in $(\mathbb{1 . 6})$, we obtain the term-by-term results,

$$
\begin{gathered}
\delta S_{W Z W}=-\frac{i}{\pi y} \int d^{2} z \operatorname{tr}\left(g^{-1} \partial g \bar{\partial} \lambda-\bar{\partial} g g^{-1} \partial \bar{\lambda}\right) \\
\delta\left(\frac{1}{\pi y} \int d^{2} z \operatorname{tr}\left(g \bar{A} g^{-1} A\right)\right)=\frac{1}{\pi y} \int d^{2} z \operatorname{tr}\left(g^{-1} A g \bar{\partial} \lambda+g \bar{A} g^{-1} \partial \bar{\lambda}\right) \\
\delta\left(-\frac{1}{\pi y^{2}} \int d^{2} z \alpha \tilde{L}_{\infty}^{a b} \operatorname{tr}\left(T_{a} g^{-1} D g\right) \operatorname{tr}\left(T_{b} g^{-1} D g\right)\right)=\frac{i}{\pi y} \int d^{2} z \operatorname{tr}\left(g^{-1} D g \bar{\partial} \lambda\right) \\
\delta\left(-\frac{1}{\pi y^{2}} \int d^{2} z \alpha \tilde{L}_{\infty}^{a b} \operatorname{tr}\left(T_{a} g \bar{D} g^{-1}\right) \operatorname{tr}\left(T_{b} g \bar{D} g^{-1}\right)\right)=\frac{i}{\pi y} \int d^{2} z \operatorname{tr}\left(g \bar{D} g^{-1} \partial \bar{\lambda}\right)
\end{gathered}
$$

whose sum $\delta S^{\prime}$ is zero on inspection.

It should also be emphasized that the non-linear and linear forms of the affine-Virasoro action are conformal and Diff $\mathrm{S}_{2}(K)$-invariant because $L_{\infty}^{a b}$ and $\tilde{L}_{\infty}^{a b}$ are solutions of the high-level Virasoro master equation. For example, temporarily assuming arbitrary $\tilde{L}_{\infty}^{a b}$, we obtain the extra terms in the $\bar{\lambda}$ variation of $S^{\prime}$

$$
\begin{aligned}
\delta S^{\prime} & =\frac{-2}{\pi y^{2}} \int d^{2} z \bar{\alpha} \operatorname{tr}\left(g^{-1} \bar{D} g T_{a}\right)\left(\tilde{L}_{\infty}^{a b}-2 \tilde{L}_{\infty}^{a c} G_{c d} \tilde{L}_{\infty}^{d b}\right) \partial\left[\bar{\xi} \operatorname{tr}\left(g^{-1} \bar{D} g T_{b}\right)\right] \\
& =0
\end{aligned}
$$

which vanish for the explicit solutions $\tilde{L}_{\infty}^{a b}$ in (1.6).

\section{The Doubly-Gauged Action}

So far, we have been discussing the world-sheet action of the $L$ theory, which is gauged by its commuting $K$-conjugate theory $\tilde{L}$, and we have seen that the 
spin-2 gauge field $\tilde{h}_{m n}$ of this theory is the world-sheet metric of the $\tilde{L}$ theory. Halpern and Yamron have also indicated how to incorporate the world-sheet metric of the $L$ theory,

$h_{m n} \equiv e^{-\chi}\left(\begin{array}{cc}-u \bar{u} & \frac{1}{2}(u-\bar{u}) \\ \frac{1}{2}(u-\bar{u}) & 1\end{array}\right), \quad \sqrt{-h} h^{m n}=\frac{2}{u+\bar{u}}\left(\begin{array}{cc}-1 & \frac{1}{2}(u-\bar{u}) \\ \frac{1}{2}(u-\bar{u}) & u \bar{u}\end{array}\right)$

which results in the doubly-gauged affine-Virasoro action, with two world-sheet metrics, $h_{m n}$ and $\tilde{h}_{m n}$ in (3.7). We will sketch these results quickly since the steps are analogous to those above for the singly-gauged theory.

One begins with the doubly-gauged hamiltonian [10],

$$
\begin{gathered}
H_{D}=\int d \tau d \sigma \mathcal{H}_{D} \\
\mathcal{H}_{D}=\frac{1}{2 \pi}\left[\left(u L_{\infty}+v \tilde{L}_{\infty}\right)^{a b} J_{a} J_{b}+\left(\bar{u} L_{\infty}+\bar{v} \tilde{L}_{\infty}\right)^{a b} \bar{J}_{a} \bar{J}_{b}\right] \equiv u \cdot T+v \cdot K
\end{gathered}
$$

which reduces to the hamiltonian (3.1) of the $L$ theory when $u=\bar{u}=1$. Defining the matrices,

$$
\begin{array}{r}
W_{a}^{b} \equiv \delta_{a}^{b}+\alpha \tilde{P}_{a}^{b}+\beta P_{a}^{b}, \quad\left(W^{-1}\right)_{a}{ }^{b}=\delta_{a}{ }^{b}+\frac{1}{2}(v-1) \tilde{P}_{a}^{b}+\frac{1}{2}(u-1) P_{a}{ }^{b} \\
\bar{W}_{a}^{b} \equiv \delta_{a}^{b}+\bar{\alpha}_{a} \tilde{P}^{b}+\bar{\beta} P_{a}^{b}, \quad\left(\bar{W}^{-1}\right)_{a}{ }^{b}=\delta_{a}{ }^{b}+\frac{1}{2}(\bar{v}-1) \tilde{P}_{a}{ }^{b}+\frac{1}{2}(\bar{u}-1) P_{a}{ }^{b} \\
\alpha=\frac{1-v}{1+v}, \quad \bar{\alpha}=\frac{1-\bar{v}}{1+\bar{v}}, \quad \beta \equiv \frac{1-u}{1+u}, \quad \bar{\beta} \equiv \frac{1-\bar{u}}{1+\bar{u}}
\end{array}
$$

we follow the prescription in (4.2) to construct the modified hamiltonian density,

$$
\begin{aligned}
\mathcal{H}_{D}^{\prime}= & \mathcal{H}_{W Z W} \\
& -\frac{1}{\pi}\left(\alpha \tilde{L}_{\infty}+\beta L_{\infty}\right)^{a b} B_{a} B_{b}-\frac{1}{2 \pi}\left(B_{a}-J_{a}\right) G^{a b}\left(B_{b}-J_{b}\right) \\
& -\frac{1}{\pi}\left(\bar{\alpha} L_{\infty}+\bar{\beta} \tilde{L}_{\infty}\right)^{a b} \bar{B}_{a} \bar{B}_{b}-\frac{1}{2 \pi}\left(\bar{B}_{a}-\bar{J}_{a}\right) G^{a b}\left(\bar{B}_{b}-\bar{J}_{b}\right)
\end{aligned}
$$

where $B$ and $\bar{B}$ are the first set of auxiliary connections. The linearized forms of the doubly-gauged action are then obtained by the simple substitution

$$
\alpha \tilde{L}_{\infty}^{a b} \rightarrow \alpha \tilde{L}_{\infty}^{a b}+\beta L_{\infty}^{a b}, \quad \bar{\alpha} \tilde{L}_{\infty}^{a b} \rightarrow \bar{\alpha} \tilde{L}_{\infty}^{a b}+\bar{\beta} L_{\infty}^{a b}
$$

in the singly-gauged actions (4.4), (4.6), or (4.7). 
Similarly, we we may integrate out the auxiliary connections of the linearized forms to obtain the non-linear form of the doubly-gauged action,

$$
\begin{aligned}
& S_{D}=\int d \tau d \sigma\left(\mathcal{L}_{D}+\Gamma\right) \\
& \mathcal{L}_{D}=-\frac{1}{8 \pi} G_{a b}\left(e_{\tau}^{a} e_{\tau}^{b}-e_{\sigma}^{a} e_{\sigma}^{b}\right)-\frac{1}{8 \pi} E^{A}\left(C^{-1}\right)_{A}{ }^{B} E_{B}
\end{aligned}
$$

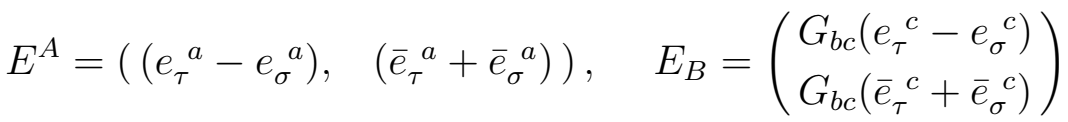

$$
\begin{aligned}
& C=\left(\begin{array}{cc}
\alpha \tilde{P}+\beta P & 1 \\
1 & \omega(\bar{\alpha} \tilde{P}+\bar{\beta} P) \omega^{-1}
\end{array}\right) \\
& C^{-1}=\left(\begin{array}{cc}
-\omega(\bar{\alpha} \tilde{P}+\bar{\beta} P) \omega^{-1} f & 1+\omega(\bar{\alpha} \tilde{P}+\bar{\beta} P) \omega^{-1} f(\alpha \tilde{P}+\beta P) \\
f & -f(\alpha \tilde{P}+\beta P)
\end{array}\right) \\
& f \equiv\left[1-(\alpha \tilde{P}+\beta P) \omega(\bar{\alpha} \tilde{P}+\bar{\beta} P) \omega^{-1}\right]^{-1}
\end{aligned}
$$

It is not difficult to check that this result agrees with the original form (3.4) of the affine-Virasoro action when $u=\bar{u}=1$ so that $\beta=\bar{\beta}=0$.

The doubly-gauged actions are invariant under Diff $\mathrm{S}_{2}(T) \times \operatorname{Diff}_{2} \mathrm{~S}_{2}(K)$, which is generated by all four stress tensors in (3.2). The Diff $\mathrm{S}_{2}(T)$ transformations of the new Diff $\mathrm{S}_{2}(T)$ gauge fields are

$$
\begin{array}{cl}
\delta u=\dot{\kappa}+\kappa \stackrel{\leftrightarrow}{\partial}_{\sigma} u, & \delta \bar{u}=\dot{\bar{\kappa}}+\bar{u} \overleftrightarrow{\partial}_{\sigma} \bar{\kappa} \\
\delta \beta=-\bar{\partial} \zeta+\zeta \stackrel{\leftrightarrow}{\partial} \beta, & \delta \bar{\beta}=-\partial \bar{\zeta}+\bar{\zeta} \stackrel{\leftrightarrow}{\bar{\partial}} \bar{\beta} \\
\zeta=(1+\beta) \kappa, & \bar{\zeta}=(1+\bar{\beta}) \bar{\kappa}
\end{array}
$$

which is a copy of eqs.(3.68), (5.2b), and (5.3), in terms of the new Diff $\mathrm{S}_{2}(T)$ gauge parameters $\kappa, \bar{\kappa}$ and $\zeta, \bar{\zeta}$. The transformations of $x, g$, and the connections $A, \bar{A}$ or $B, \bar{B}$ may be obtained by the substitution

$$
\lambda^{a} \rightarrow \lambda^{a}=2\left(\xi \tilde{L}_{\infty}^{a b}+\zeta L_{\infty}^{a b}\right) B_{b}, \quad \bar{\lambda}^{a} \rightarrow \bar{\lambda}^{a}=2\left(\bar{\xi} \tilde{L}_{\infty}^{a b}+\bar{\zeta} L_{\infty}^{a b}\right) \bar{B}_{b}
$$

for the local Lie $g \times$ Lie $g$ gauge parameters $\lambda, \bar{\lambda}$ in eqs. (5.2), (5.4), and (5.5). The forms in (6.8) show that Diff $\mathrm{S}_{2}(T) \times$ Diff $\mathrm{S}_{2}(K)$ is also embedded in (classical) affine $G \times G$. 


\section{Conclusions}

Some time ago, Halpern and Yamron found the generic affine-Virasoro action, which is a Lorentz, conformal, and Diff $\mathrm{S}_{2}$-invariant world-sheet action for the generic irrational conformal field theory. This action exhibits an elegant underlying geometry associated with the embedding of Diff $\mathrm{S}_{2}$ in (classical) affine $G \times G$, but the form of the action is highly non-linear.

In this paper, we introduced auxiliary fields $A_{a}, \bar{A}_{a}, a=1, \ldots, \operatorname{dim} g$ to find the linearized form of the affine-Virasoro action, given in eq.44.7). The embedding of Diff $\mathrm{S}_{2}$ in (classical) affine $G \times G$ plays a particularly transparent role in the linearized action, which is clearly seen as a Diff $\mathrm{S}_{2}$-gauged WZW model. In particular, the auxiliary fields $A, \bar{A}$ transform under Diff $\mathrm{S}_{2}$ as local Lie $g \times$ Lie $g$ connections, which accounts for the intriguing resemblance of the linearized action to the usual (Lie algebra) gauged WZW model.

A next step is to integrate out the spin-2 gauge fields of the non-linear or the linearized action, following the usual semiclassical development in twodimensional gravity. In this case, we are gauging a $K$-conjugate "matter" system with semiclassical central charge $c\left(\tilde{L}_{\infty}\right)=\operatorname{dim} \tilde{P}$, so we expect the semiclassical action to have the form [21]

$$
\begin{gathered}
S=S_{W Z W}+S_{G}+S_{F F} \\
c\left(L_{g, \infty}\right)=\operatorname{dim} g, \quad c_{G}=-26, \quad c_{F F}=1-12 Q^{2}=26-c\left(\tilde{L}_{\infty}\right)
\end{gathered}
$$

in the WZW gauge $\alpha=\bar{\alpha}=0$. Here, $S_{G}$ is the action of the diffeomorphism

ghosts and $S_{F F}$ is a Feigen-Fuchs action with its background charge adjusted so that the gauged subsystem ( $K$-conjugate matter + ghosts + Feigen-Fuchs) has zero semiclassical central charge. Then we may verify that the total semiclassical central charge of the theory is the semiclassical central charge of the $L$ theory,

$$
c(\text { total })=c\left(L_{g, \infty}\right)+c_{G}+c_{F F}=c\left(L_{g, \infty}\right)-c\left(\tilde{L}_{\infty}\right)=c\left(L_{\infty}\right)=\operatorname{dim} P
$$

as it should be.

A full quantum version of this picture may be realized with standard BRST operators of the form,

$$
Q=\oint \frac{d z}{2 \pi i} c\left(\tilde{T}+T_{F F}+\frac{1}{2} T_{G}\right), \quad c_{F F}=26-c(\tilde{L})
$$


where $\tilde{T}=T(\tilde{L})$ is the quantum stress tensor of the $\tilde{L}$ theory, with quantum central charge $c(\tilde{L})$. Up to discrete states, these BRST operators reproduce the physical state conditions (1.5) for the generic theory $L$.

After completion of this work, we received a paper by Tseytlin [20] which studies an ungauged but presumably related action with chiral group variables and $\operatorname{dim} g$ auxiliary fields. The hamiltonian of this action is the ungauged basic affine-Virasoro hamiltonian $H_{0}$ in (3.1), with a different canonical representation of the currents, so the gauged form of this action should be equivalent to the results presented here.

\section{Acknowledgments}

We thank M. W. Craig, who participated in the early states of this work.

The work of MBH was supported in part by the Director, Office of Energy Research, Office of High Energy and Nuclear Physics, Division of High Energy Physics of the U.S. Department of Energy under Contract DE-AC03-76SF00098 and in part by the National Science Foundation under grant PHY90-21139. The work of JdB was supported in part by the National Science Foundation under grant PHY93-09888. 


\section{References}

[1] V.G. Kac, Funct. Anal. App. 1 (1967) 328; R.V. Moody, Bull. Am. Math. Soc. 73 (1967) 217.

[2] K. Bardakçi and M.B. Halpern, Phys. Rev. D3 (1971) 2493.

[3] M.B. Halpern and E. Kiritsis, Mod. Phys. Lett. A4 (1989) 1373; Erratum ibid. A4 (1989) 1797.

[4] A.Yu Morozov, A.M. Perelomov, A.A. Rosly, M.A. Shifman and A.V. Turbiner, Int. J. Mod. Phys. A5 (1990) 803.

[5] M.B. Halpern, "Recent Developments in the Virasoro Master Equation", in the proceedings of the Stony Brook conference, Strings and Symmetries 1991, World Scientific, 1992.

[6] M.B. Halpern, "Recent Progress in Irrational Conformal Field Theory", Berkeley preprint, UCB-PTH-93/25, hep-th 9309087, 1993. To appear in the proceedings of the Berkeley conference, Strings 1993.

[7] M.B. Halpern and N.A. Obers, Int. J. Mod. Phys. A9 (1994) 265.

[8] M.B. Halpern and N.A. Obers, Int. J. Mod. Phys. A9 (1994) 419.

[9] M.B. Halpern and N.A. Obers, "Flat Connections and Non-Local Conserved Quantities in Irrational Conformal Field Theory", Berkeley preprint, UCBPTH-9/33, hep-th 9312050.

[10] M.B. Halpern and J.P. Yamron, Nucl. Phys. B351 (1991) 333.

[11] K. Bardakci, E. Rabinovici and B. Säring, Nucl. Phys. B299 (1988) 157; K. Gawedzki and A. Kupiainen, Phys. Lett. B215 (1988) 119; Nucl. Phys. B320 (1989) 625.

[12] D. Karabali, Q-Han Park, H.J. Schnitzer and Z. Yang, Phys. Lett. B216 (1989) 307; D. Karabali and H.J. Schnitzer, Nucl. Phys. B329 (1990) 649.

[13] M.B. Halpern, Phys. Rev. D4 (1971) 2398. 
[14] P. Goddard, A. Kent and D. Olive, Phys. Lett. B152 (1985) 88.

[15] E. Witten, Comm. Math. Phys. 92 (1984) 455; G. Segal, unpublished.

[16] V.G. Knizhnik and A.B. Zamolodchikov, Nucl. Phys. B247 (1984) 83.

[17] M.B. Halpern and N.A. Obers, Nucl. Phys. B345 (1990) 607.

[18] M.B. Halpern and N.A. Obers, J. Math. Phys. 33 (1992) 3274.

[19] P. Bowcock, Nucl. Phys. B316 (1989) 80.

[20] A.A. Tseytlin, "On a 'Universal' Class of WZW-Type Conformal Models", CERN-TH.7068/93, hep-th/9311062, 1993.

[21] J. Distler and H. Kawai, Nucl. Phys. B321 (1989) 509; N.E. Mavromatos and J.L. Miramontes, Mod. Phys. Lett. A4 (1989) 1847; E. D'Hoker and P.S. Kurzepa, Mod. Phys. Lett. A5 (1990) 1411. 